\title{
VISCOUS DARK COSMOLOGY WITH ACCOUNT OF QUANTUM EFFECTS
}

\author{
I. BREVIK 1 and O. GORBUNOVA2 \\ Department of Energy and Process Engineering, Norwegian University of \\ Science and Technology, N-7491 Trondheim, Norway
}

\begin{abstract}
The analytic properties of the energy density $\rho(t)$ of the cosmic fluid, and the Hubble parameter $H(t)$, are investigated near to the future singularity $t=t_{s}$ assuming different forms for the equation of state. First, it is shown that the inclusion of quantum effects coming from the conformal anomaly modifies the singularity. Thereafter, we consider the effect coming from a bulk viscosity in the fluid. The viscosity tends to reduce the magnitude of $t_{s}$, but does not alter the singularity itself (the exponent). Main emphasis is laid on the simple case when the equation of state is $p=w \rho$, with $w$ a constant.
\end{abstract}

\section{Introduction}

It is actually not more than about five years since the possible occurrence of a singularity of the universe in the far future - usually called the Big Rip - was first discussed in the literature [1, 2, 3]. To begin with, two different types of singularities were investigated. Later, it became clear that there are two possibilities more, so that present we know about four different types. For convenience, we quote from Ref. [5] the following classification:

(i) Type I ("Big Rip"): For $t \rightarrow t_{s}, a \rightarrow \infty, \rho \rightarrow \infty$, and $|p| \rightarrow \infty$, or $p$ and $\rho$ are finite at $t=t_{s}$.

(ii) Type II ("sudden"): For $t \rightarrow t_{s}, a \rightarrow a_{s}, \rho \rightarrow \rho_{s}$, and $|p| \rightarrow \infty$,

(iii) Type III: For $t \rightarrow t_{s}, a \rightarrow a_{s}, \rho \rightarrow \infty$, and $|p| \rightarrow \infty$,

(iv) Type IV: For $t \rightarrow t_{s}, a \rightarrow a_{s}, \rho \rightarrow 0,|p| \rightarrow 0$, or $p$ and $\rho$ are finite. Higher order derivatives of $H$ diverge.

\footnotetext{
${ }^{1}$ E-mail: iver.h.brevik@ntnu.no

${ }^{2}$ On leave from Tomsk State Pedagogical University, Tomsk, Russia. Email: gorbunovaog@yandex.ru.
} 
Here the notation is standard, $a$ meaning the scale factor and $t_{s}$ referring to the instant of the singularity. These theories assume the cosmic fluid to be ideal, i.e. nonviscous.

As often happens in physics, when the mathematical formalism encounters singularities, this indicates that the description has been too simplistic. It becomes natural to ask: can the singularity be modified or softened by drawing other physical effects into consideration? The answer turns out to be affirmative, due to at least two reasons. The first is the effect of quantum corrections due to the conformal anomaly. It turn up in various cosmological applications: dynamical Casimir effect with conformal anomaly [8], or dark fluid with conformal anomaly [9]. Explicit calculation shows this anomaly to cause a softening of the singularity [5, 10]. A second reason - the one to be treated in the present paper - is to take into account the bulk viscosity of the cosmic fluid. From a hydromechanical standpoint a generalization of the cosmic theory so as to encompass viscosity is most natural. As we will see, the viscosity also acts in the direction of softening. Viscous cosmology, beginning with the seminal paper of Misner [11] seems generally to have attracted increased interest in recent years. Some references in this direction are [12, 13, 14, 15, 16, 17, 18].

For convenience, and for reference purposes, we give in the next section a brief overview of the singularity application of conformal anomaly theory. Thereafter, from Section 3 onwards we embark on the core of the present paper, namely to include a bulk viscosity in the Einstein equations.

\section{Quantum effects, when $p=-\rho-A \rho^{\beta}$.}

We shall content ourselves by considering specific examples. Close to the borderline between the quintessence and phantom regions, the equation-ofstate (EOS) parameter $w=p / \rho$ is near to -1 . One natural choice for the equation of state is the following [4, [5, 6, 7]:

$$
p=-\rho-A \rho^{\beta}
$$

where $A$ and $\beta$ are constants. Thus $p$ always diverges when $\rho$ becomes infinite. Note that this may be considered as a viscous dark energy.

We shall distinguish between two cases, viz. $1 / 2<\beta \leq 1$, and $\beta>1$. They may be classified as follows, referring to the notation in the previous section: 
(1) $1 / 2<\beta \leq 1$ : The singularity is of Type I. The dominant energy condition (DEC), requiring $\rho \geq 0, \rho \pm p \geq 0$, is broken. The EOS parameter approaches -1 for either sign of $A$, when $\rho \rightarrow \infty$. This case implies that $\rho$ diverges in a finite future. One obtains [5]

$$
\rho \sim\left(t_{s}-t\right)^{\frac{2}{1-2 \beta}}, \quad H \sim\left(t_{s}-t\right)^{\frac{1}{1-2 \beta}},
$$

with $t_{s}$ the singularity time and $H=\dot{a} / a$ the Hubble parameter.

(2) $\beta>1$ : The singularity is of Type III. The DEC is also now broken. The magnitude of the EOS parameter goes to infinity, i.e., $w \rightarrow+\infty(-\infty)$ for $A<0(A>0)$. Equations (2) still hold.

We shall be concerned mainly with the case $\beta>1$ in this section.

Consider next the quantum contribution to the conformal anomaly. The complete energy density is $\rho_{t o t}=\rho+\rho_{A}$. Taking the trace of the conformal anomaly energy-momentum tensor, $T_{A}=-\rho_{A}+3 p_{A}$, plus observing the energy conservation law,

$$
\dot{\rho}_{A}+3 H\left(\rho_{A}+p_{A}\right)=0,
$$

we find that

$$
p_{A}=-4 \rho_{A}-\dot{\rho}_{A} / H .
$$

Thus we obtain for the conformal anomaly energy density [5]

$$
\begin{gathered}
\rho_{A}=-\frac{1}{a^{4}} \int d t a^{4} H T_{A} \\
=-\frac{1}{a^{4}} \int d t a^{4} H\left[-12 b \dot{H}^{2}+24 b^{\prime}\left(-\dot{H}^{2}+H^{2} \dot{H}+H^{4}\right)\right. \\
\left.-\left(4 b+6 b^{\prime \prime}\right)\left(\dddot{H}+7 H \ddot{H}+4 \dot{H}^{2}+12 H^{2} \dot{H}\right)\right],
\end{gathered}
$$

where $b, b^{\prime}$ and $b^{\prime \prime}$ are constants, occurring in the expression for the conformal trace anomaly,

$$
T_{A}=b\left(F+\frac{2}{3} \square R\right)+b^{\prime} G+b^{\prime \prime} R .
$$

Here $F$ is the squared Weyl tensor and $G$ the Gauss-Bonnet invariant. Explicitly, if there are $N$ scalars, $N_{1 / 2}$ spinors, $N_{1}$ vectors, $N_{2}$ gravitons, and $N_{H D}$ higher derivative conformal scalars, one has for $b$ and $b^{\prime}$ the expressions

$$
b=\frac{N+6 N_{1 / 2}+12 N_{1}+611 N_{2}-8 N_{H D}}{120(4 \pi)^{2}},
$$




$$
b^{\prime}=\frac{N+11 N_{1 / 2}+62 N_{1}+1411 N_{2}-28 N_{H D}}{360(4 \pi)^{2}},
$$

whereas $b^{\prime \prime}$ is an arbitrary constant whose value depends on the regularization.

The quantum corrected FRW equation is, with $\kappa^{2}=8 \pi G$,

$$
\frac{3}{\kappa^{2}} H^{2}=\rho+\rho_{A} .
$$

As $R$ is large near $t=t_{s}$, we assume $\left(3 / \kappa^{2}\right) H^{2} \ll\left|\rho_{A}\right|$. Hence, $\rho \sim-\rho_{A}$.

In the presence of quantum effects, suppose that

$$
\rho=\rho_{0}\left(t_{s}-t\right)^{\tilde{\gamma}}
$$

as in Ref. [5]. From the energy conservation equation

$$
H=\frac{\dot{\rho}}{3 A(\rho)^{\beta}} \simeq-\frac{\tilde{\gamma} \rho_{0}^{1-\beta}}{3 A}\left(t_{s}-t\right)^{-1+\tilde{\gamma}(1-\beta)}
$$

we get, by taking the most singular term, $\dot{\rho} \sim-\left(4 b+6 b^{\prime \prime}\right) H \dddot{H}$. Integrating with respect to $t$ we get $\rho$ to be of the form (9), with $\tilde{\gamma}=4 /(1-2 \beta)$. Thus

$$
\rho \sim\left(t_{s}-t\right)^{\frac{4}{1-2 \beta}}, \quad H \sim\left(t_{s}-t\right)^{\frac{3-2 \beta}{1-2 \beta}}
$$

near $t=t_{s}$. If we compare this with the case of no quantum corrections, we see that the energy density diverges more rapidly in the present case, whereas $H$ becomes less singular. That means, the quantum corrections moderate the singularity. Note that

$$
\frac{H^{2}}{\rho} \sim\left(t_{s}-t\right)^{\frac{2-4 \beta}{1-2 \beta}} \rightarrow 0,
$$

showing that our approximation neglecting the left hand side of Eq. (8) is consistent.

The case $\beta>1$ as assumed here, corresponds to singularity of Type III. The same properties may be found for the Big Rip (Type I) singularity.

\section{Viscosity-generated singularity, when $p=$ $-\rho-f(\rho)$}

Assume now that the cosmic fluid is viscous, with a bulk viscosity $\zeta$. In general, $\zeta=\zeta(\rho)$. Hereafter we leave out the conformal anomaly, so that $\rho_{A}=0$. 
The FRW equation becomes accordingly $\left(3 / \kappa^{2}\right) H^{2}=\rho$, where the matter energy density $\rho$ incorporates the modification due to the viscosity. We assume the cosmic fluid to be thermodynamically a simple, one-component, fluid. We start by assuming the general form $p=-\rho-f(\rho)$, with $f(\rho)$ a general function.

This case was analyzed in Ref. [19]. The essential difference from the foregoing is that the energy conservation equation is now

$$
\dot{\rho}+3 H(\rho+p)=9 \zeta H^{2}
$$

(note that the scalar expansion is $\theta=3 H$ ). We let $t=0$ denote present time, and let subscript 0 refer to present time quantities. The viscosity-generated singularity time is denoted as $t_{s \zeta}$. From Eq. (9) in [19] we have

$$
t=\frac{1}{\sqrt{3} \kappa} \int_{\rho_{0}}^{\rho} \frac{d \rho}{\sqrt{\rho} f(\rho)[1+\kappa \zeta(\rho) \sqrt{3 \rho} / f(\rho)]} .
$$

Singularities of Type I or III occur if $t=t_{s \zeta}$ corresponds to $\rho \rightarrow \infty$.

We assume henceforth that $\zeta(\rho) \rightarrow \zeta$ is a constant, and consider two specific choices for the function $f(\rho)$.

\section{The case when $f(\rho)=\alpha \rho$}

We consider this case first because it is simplest. It corresponds to an equation of state in the form

$$
p=w \rho=-(1+\alpha) \rho,
$$

with $\alpha$ a constant. From Eq. (17) in [19] we have

$$
H(t)=\frac{H_{0} e^{t / t_{c}}}{1-\frac{3 \alpha}{2} H_{0} t_{c}\left(e^{t / t_{c}}-1\right)},
$$

where $t_{c}$ is a 'viscosity time',

$$
t_{c}=\left(\frac{3}{2} \kappa^{2} \zeta\right)^{-1}
$$

It is convenient to introduce two new nondimensional quantities $x$ and $K$,

$$
x=\frac{t_{s \zeta}}{t_{c}}, \quad K=\frac{2}{3 \alpha H_{0} t_{s \zeta}} .
$$


From Eq. (15) it is seen that $H$ becomes infinite when

$$
x=\ln (1+K x) .
$$

This transcendental equation for $x$ can have two solutions.

(i) Nonviscous solution. There is always the solution $x=0$, corresponding to $t_{c}=\infty$ or $\zeta=0$. This is accordingly the solution for a nonviscous fluid. We will call the singularity time in this case $t_{s 0}$. We get

$$
t_{s 0}=\frac{2}{3 \alpha H_{0}} .
$$

It is desirable to write the solutions such that the difference from the singularity time occurs explicitly. First, we have

$$
H(t)=\frac{H_{0} t_{s 0}}{t_{s 0}-t},
$$

which corresponds to

$$
a(t)=\frac{a_{0} t_{s 0}^{2 / 3 \alpha}}{\left(t_{s 0}-t\right)^{2 / 3 \alpha}} .
$$

Next, as $H^{2} \propto \rho$ according to the FRW equation, we get

$$
\rho(t)=\frac{\rho_{0} t_{s 0}^{2}}{\left(t_{s 0}-t\right)^{2}} .
$$

Comparing Eqs. (20) and (22) with Eq. (22), we see that corresponding equations for $H(t)$ and $\rho(t)$ are of the same form, if $\beta=1$. This is as we might expect, as the equation of state in the present case corresponds to $A=\alpha, \beta=1$. The singularity is of Type I if $\alpha>0$, and of type III if $\alpha<0$.

(ii) Viscous solution. In the viscous case, $x>1$, Eq. (18) has a second root if the slope $K$ is larger than one. As $K$ is actually the same as the ratio $t_{s 0} / t_{s \zeta}$, we thus get the following condition for solution

$$
t_{s \zeta}<t_{s 0} .
$$

The presence of a bulk viscosity thus reduces the future singularity time. It would not be so easy to foresee this property beforehand.

For a given value of $t_{c}$, the transcendental equation (18) does not allow us to solve for $t_{s \zeta}$ explicitly. However, we may easily handle analytically the 
limiting case $t \rightarrow t_{s \zeta}$, which is the situation of main interest here. Using the approximation $e^{\tau / t_{c}}=1+\tau / t_{c}$ with $\tau=t_{s \zeta}-t$, we obtain

$$
H(t) \rightarrow \frac{H_{0} t_{s 0}}{t_{s \zeta}-t}, \quad t \rightarrow t_{s \zeta}
$$

This formula holds for all values of $\zeta$.

It is thus apparent that we get the same singular behavior of $H(t)$ in the viscous case as we did previously, in Eqs.(20) and (2) (the latter under the assumption that $\beta=1$ ). Correspondingly, we get in the present case

$$
\begin{gathered}
a(t) \sim\left(t_{s \zeta}-t\right)^{-2 / 3 \alpha}, \quad t \rightarrow t_{s \zeta} \\
\rho(t) \sim\left(t_{s \zeta}-t\right)^{-2}, \quad t \rightarrow t_{s \zeta} .
\end{gathered}
$$

Whereas the viscosity tends to shorten the future singularity time, it does not modify the exponents in the singularity. Again, the singularity is of Type I if $\alpha>0$, and of type III if $\alpha<0$.

When dealing with viscosity we have introduced two different times, namely a viscosity time $t_{c}$ defined in Eq. (16), and a singularity time $t_{s \zeta}$. One may wonder: can anything be said about the relative magnitude of these times? As most cosmological theories are formulated without viscosity, it is natural to assume that the cases of main physical interest pertain to setting $\zeta$ very small, or $t_{c}$ very large. Accordingly, the quantity $x$ defined in Eq. (17) should be expected to be small, corresponding to $t_{s \zeta} \ll t_{c}$.

Another related point is to inquire whether there is a relationship between the singularity time $t_{s}$ with or without conformal anomaly considered in Section 2, and the viscosity singularity time $t_{s \zeta}$. Although there does not seem to be a close relationship, it is in our opinion most natural to identify the nonviscous time $t_{s 0}$ in Eq. (19) with the previous time $t_{s}$. As we have seen, the effect from bulk viscosity is to reduce the singularity time. We thus suggest that the relationship

$$
t_{s \zeta}<t_{s}
$$

is quite general.

Note that one may take into account quantum effects in this case too. However, the corresponding equations are very cumbersome and require numerical study. Such a study indicates that that quantum effects may again soften the future singularity. 


\section{Remarks on the case when $f(\rho)=A \rho^{\beta}$}

In this more general case we go back to Eq. (13) (still assuming that $\zeta$ is constant). Leaving at first the integration limits unspecified, we have

$$
t=\frac{1}{3 \kappa^{2}} \frac{1}{\zeta} \int \frac{d \rho}{\rho\left[1+\frac{A}{\sqrt{3} \kappa \zeta} \rho^{\beta-1 / 2}\right]} .
$$

As $\beta>1 / 2$, it is seen that the last term in the denominator (the viscosity term) dominates for large $\rho$. We perform the integration from an initial value of $\rho$ already lying in this region, up to $\rho \rightarrow \infty$. The corresponding time integration limits are $t$ and $t_{s \zeta}$. We obtain approximatively

$$
t_{s \zeta}-t=\frac{1}{\sqrt{3} \kappa} \frac{1}{A} \frac{1}{\beta-1 / 2} \frac{1}{\rho^{\beta-1 / 2}} .
$$

Thus

$$
\rho(t) \sim\left(t_{s \zeta}-t\right)^{\frac{2}{1-2 \beta}}, \quad t \rightarrow t_{s \zeta}
$$

which generalizes Eq. (26) and reduces to it in the case when $\beta=1$. Equation (30) has actually the same form as Eq. (22).

\section{Summary}

We may summarize our results as follows.

1) We started out by giving a brief overview of the four types of future singularities, listing in Eq. (2) the known forms for $\rho(t)$ and $H(t)$ in the case where the equation of state is assumed as in Eq. (1). Actual in our context were singularities of Type I and Type III.

2) Inclusion of quantum effects coming from the conformal anomaly - cf. Eq. (5) - caused the exponents of the future singularity to be modified.

3) In Section 3 we left out quantum effects, but considered instead the influence from a bulk viscosity $\zeta$. Assuming the simple form (14) for the equation of state, we showed in Eqs. (24)-(26) that the viscosity acts so as to shorten the singularity time, but it does not change the nature of the singularity. The same property was found when dealing with a more complicated equation of state in Section 5. The explicit calculations were limited to the case when $\zeta$ was a constant. 
Finally we mention that, under quite general conditions, preliminary numerical work on quantum effects indicates that they tend to soften the future singularity.

\section{Acknowledgment}

The work of O. G. was supported in part by RFBR grant 06-01-00609 and LRSS project N.2553.2008.2.

\section{References}

[1] R. R. Caldwell, M. Kamionkowski and N. N. Weinberg, Phys. Rev. Lett. 91, 071301 (2003).

[2] B. McInnes, J. High Energy Phys. 0208, 029 (2002).

[3] J. D. Barrow, Class. Quantum Grav. 21, L79 (2004).

[4] S. Nojiri and S. D. Odintsov, Phys. Rev. D 70, 103522 (2004).

[5] S. Nojiri, S. D. Odintsov and S. Tsujikawa, Phys. Rev. D 71, 063004 (2005).

[6] S. Nojiri and S. D. Odintsov, Phys. Rev. D 72, 0123003 (2005).

[7] S. Nojiri and S. D. Odintsov, Int. J. Geom. Meth. Mod. Phys. 4, 115 (2007).

[8] I. Brevik and S. D. Odintsov, Phys. Lett. B 455, 104 (1999); I. Brevik, K. Milton, S. D. Odintsov and K. Osetrin, Phys. Rev. D 62, 064005 (2000).

[9] S. Nojiri and S. D. Odintsov, Phys. Lett. B 562, 147 (2003).

[10] S. Nojiri and S. D. Odintsov, Phys. Lett. B 595, 1 (2004); E. Elizalde, S. Nojiri and S. D. Odintsov, Phys. Rev. D 70, 043539 (2004).

[11] C. W. Misner, Astrophys. J. 151, 431 (1968).

[12] T. Padmanabhan and S. M. Chitre, Phys. Lett. A 120, 433 (1987). 
[13] Ø. Grøn, Astrophys. Space Sci. 173, 191 (1990).

[14] I. Brevik and A. Hallanger, Phys. Rev. D 69, 024009 (2004).

[15] J. Ren and X. H. Meng, Phys. Lett. B 633, 1 (2006).

[16] S.Capozziello, V. F. Cardone, E. Elizalde, S. Nojiri and S. D. Odintsov, Phys. Rev. D 73, 043512 (2006).

[17] I. Brevik, Gen. Rel. Grav. 38, 1317 (2006).

[18] R. Sussman, arXiv:0801.3324 [gr-qc].

[19] I. Brevik and O. Gorbunova, Gen. Rel. Grav. 37, 2039 (2005). 